\title{
DESIGN OF MECHANICAL COMPACTOR FOR PET BOTTLES
}

\author{
Eng. Master Gabriel Cristian POROSCHIANU ${ }^{1}$ \\ PhD. Lecturer Georgeta HARAGA ${ }^{1}$ \\ ${ }^{1}$ "Politehnica" University of Bucharest
}

\begin{abstract}
The paper is focused on the process of compaction of PET bottles to reduce the pollution that is caused by them. In the first part of the paper there is a brief introduction about PET and the necessity of reducing the space that PET bottles occupy when they are stored before the recycling process. In the second part of the paper is presented the design of a mechanical compactor for PET bottles, made of aluminium, designed to be used in every household.
\end{abstract}

KEYWORDS: process of compaction, PET, mechanical compactor, recycling

\section{INTRODUCTION}

Plastic is one of the materials that has more "lives" and can be recycled for several times. Our paper puts forward a principle of sustainable development for a social, economic and environmental policy, as well as the benefits in all three directions.

Nowadays, it is envisaged to obtain products with a long life cycle, which correspond best to their own goal, requiring very low consumption for their achievement, but also for maintenance or operation, without endangering the environment [1].

The United Nations Environment Program estimates that, on average, on every square kilometer of all the oceans in the world are floating nearly 18,000 such pieces of plastic - most of them made of PET [9]. Because this material is obtained from the processing of crude oil, it consumes a precious resource. Every year, only the PET packaging industry consumes over 150 billion liters of crude oil, enough to provide gas for half a million cars for a full year. According to the latest European Commission/EC/ published in the Generation Awake campaign, a European waste reduction and recycling initiative, more than $60 \%$ of Romanians would like to have more waste recycling and composting facilities in the area where they live. By customizing PET packaging, our country empties about 3 billion pieces annually, or about 140 thousand tons, of which over $90 \%$ come from individuals [4]. This quantity increases by about $20 \%$ each year. So solving environmental pollution problems with nondegradable PET bottles is also a must for our city. PET was first synthesized in North America in the mid-40s by DuPont chemist researchers.
In the late 1950s, researchers found a way to stretch PET into thin films. As technology advances, scientists have found new ways to blow PET bottles. Under these circumstances, PET bottle was patented in 1973 and was first recycled in 1977 [5].

PET is abbreviated from polyethylene terephthalate and comes in the form of a resin (a polyesterthermoplastic). Polyethylene terephthalate is a combination of two monomers: modified ethylene glycol and purified terephthalic acid. It has become a widespread material in the food industry (bottling of soft drinks, water, dairy, oil, vinegar, etc.), being cheap, lightweight, shock resistant, recyclable. PET packaging, like all plastic materials, is not biodegradable, persisting in storage locations for hundreds of years. In addition, the unsightly nature of PET recipients that ruin the appearance of natural spaces and which, by their large volume, aggravate any landscape, should not be omitted. PET (polyethylene terephthalate) is an extremely resistant material. In nature, it takes about $700-800$ years until it begins to decay and completely disintegrates only after about 1.000 years [5, 9]. (fig.1)

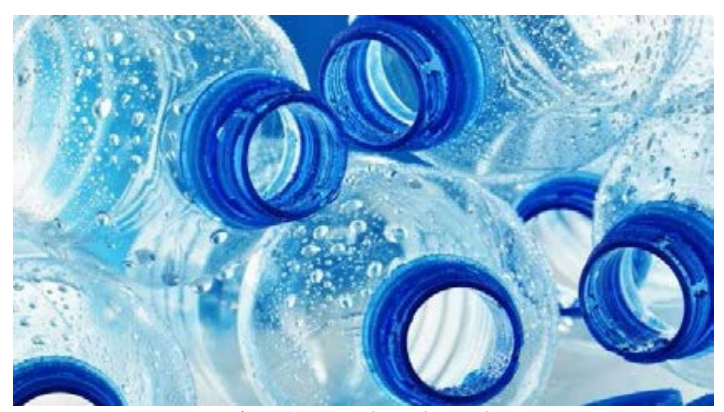

Fig. 1 PET bottles [4] 
PET bottles have a very large share of the world's use, due to their advantages: low cost, low specific weight, very high maneuverability, cheap manufacturing technology.

\section{THE RECYCLING STEPS}

Although it is so resistant to degradation, PET is $100 \%$ recyclable. Moreover, there are large manufacturers of clothing that exclusively use recycled PET/polyester fibers. In this chapter, we will show what happens to the bottles after using.

The recycling steps are:

Collecting and compaction: The consumer of PET packed products no longer throws the bottle into the trash after emptying, but compacts it using the solutions below. The PET container, instead of becoming non-degradable waste, is compacted and it reaches the first recycling step, also becoming a semirecycled material.

Storage: Compact PET bottles are easily stored in raffia/plastic/paper bags pending recycling. Advantages: comfortable handling; storage space economy;

Shipment: Compacted and collected PET bottles will be shipped to the PET recycling center for recycling. Advantages: efficient transport, collected from many places;

Recycling: PET bottles are passed through a washing and grinding technology in specialized units where they have been shipped from collection centers. The product obtained, called "flakes", is a raw material for the plastics industry in the production of granules, synthetic fibers, belts, packaging, etc.

\section{Applications of the recycled PET bottles:}

PET can be used for: polyester fibers (75\%), used in turn as raw material for carpets, upholstery, toys, textiles for the textile industry, automotive industry, foil, straps, strips, new PET and non-food packaging [6].

Of the many examples of PET recycling, we mention just a few [7]:

- every ton of recycled plastic saves between 700 and $800 \mathrm{~kg}$ of crude oil;

- recycling a plastic bottle saves energy for the operation of a $60 \mathrm{~W}$ bulb for 6 hours;

- from 10 recycled PET bottles, one can produce $1 \mathrm{t}$ shirt or $1 \mathrm{~m}^{2}$ of carpet.

- from 50 PET bottles one can make a sweater.

Physical properties of PET:

The material has good mechanical properties, very good dimensional stability, thermal behavior, reduced friction coefficient. Fiber-reinforced material gains mechanical performance, and heat resistance increases to $200{ }^{\circ} \mathrm{C}$. Polyethylene terephthalate is a good insulator, successfully used for medium and low voltages, being very poorly affected by the humidity conditions of the environment. PET has the following density: $\rho=1010 \div 1091 \mathrm{~kg} / \mathrm{m}^{3}$.

\section{THE DESIGN OF THE MECHANICAL PET COMPACTOR}

If we do not reuse PET bottles, then we have to collect them selectively. For this to be done as efficiently as possible, plastic packaging from which we drink water or juice must be compacted. Thus, these packages occupy less space in the pub and can be collected as much as possible.

Compaction is a very important step in waste recycling because it greatly reduces transport costs but also reduces the amount of space required to store waste waiting to be recycled. (fig. 2)

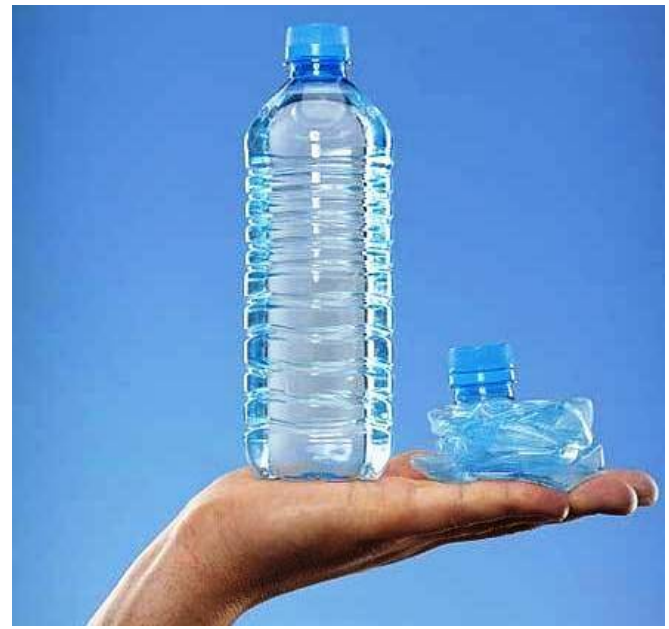

Fig. 2 Compaction of PET bottles [6]

The PET compactor contributes to the efficient storage of PET waste in terms of effort and time dedicated to this action. A type of device that addresses the problem of PET recycling is a manual mechanical compactor.

Compaction by manual deformation is not so very difficult. Given the rise in living standards, the need to have a PET compactor became necessary in every home, restaurant, school, institution, leisure place. The manual mechanical PET compactor design proposed in this paper has eight components and it is made in a graphical program called SolidWorks. This compactor can be transported easily, it can be used by any person no matter the age, has a light weight and it is made of aluminium as it can be seen in figures 3 and 4.

The physical properties of aluminium [10]:

- white-silver metal, which crystallizes in the cubic system with centered faces;

- slightly less hard metal than copper;

- very good driver of heat and electricity;

- electrical and thermal conductivity is about half as much as copper;

- aluminum is very plastic, can be rolled, beaten in very thin sheets or turned into fine wire.

The chemical properties of aluminium [10]: - chemically active element; 
- although it has a strong electropositive character at normal temperature, pure aluminium becomes stable in the air because it is protected by the thin film of aluminum oxide formed on its surface.

The formation of aluminium oxide film that protects metals from air and moisture can be prevented by amalgamation of the aluminum surface. Therefore, a piece of aluminum coated by rubbing the surface with a concentrated mercury chloride solution or mercury powder or directly with mercury, exposed to air, is covered with white efflorescence due to the formation of aluminium hydroxide.

$$
4 \mathrm{Al}+3 \mathrm{O}_{2}=2 \mathrm{Al}_{2} \mathrm{O}_{3}
$$

The amount of heat released in this reaction is significant.

Because of the high affinity of Alumina for Oxygen, it removes it from the less active metal oxides.

For example, if a mixture of aluminium powder and iron oxide is ignited, it is because of the reaction that occurs:

$$
2 \mathrm{Al}+\mathrm{Fe}_{2} \mathrm{O}_{3}=\mathrm{Al}_{2} \mathrm{O}_{3}+2 \mathrm{Fe}
$$

Description and characteristics of the mechanical compactor for PET bottles

\section{A. Description}

The PET compactor contributes to the efficient storage of PET waste, in terms of effort and time dedicated to this action.

\section{B. Characteristics and operation mode}

How does PET compactor work: Unscrew the cover as much as possible so the air comes out of the PET bottle in the compaction process. Place the PET thread in the upper hole and place it in the compactor. By lowering the handle to full compaction, the air completely exits the PET container. If the compaction process is difficult, check that you have unscrewed the cap fully. Compress the PET bottle to the maximum possible position. Secure the cover as far as possible before lifting the compactor arm to prevent the air from returning to the PET recipient.

\section{Advantages}

Among the advantages of using this type of compactor the following ones are the most important [8]:

- lack of electricity;

- a much faster compaction;

- suitable for any potential buyer due to its low cost;

- low handling time;

- minimal physical effort;

- variability of pressing plastic bottles 0,5 - 21;

- optimization of the storage space (after compaction the volume of the PET bottle is reduced by up to $20 \%$ ).

The modeling of this mechanical compactor it was made with SolidWorks software.
SolidWorks has a greater ability to impose geometric constraints at the sketch level, in that it allows for constraints applied at point level [2, 3].

\section{Disadvantages:}

- compaction by manual deformation is rather cumbersome and requires physical effort;

- high cost of aluminum.

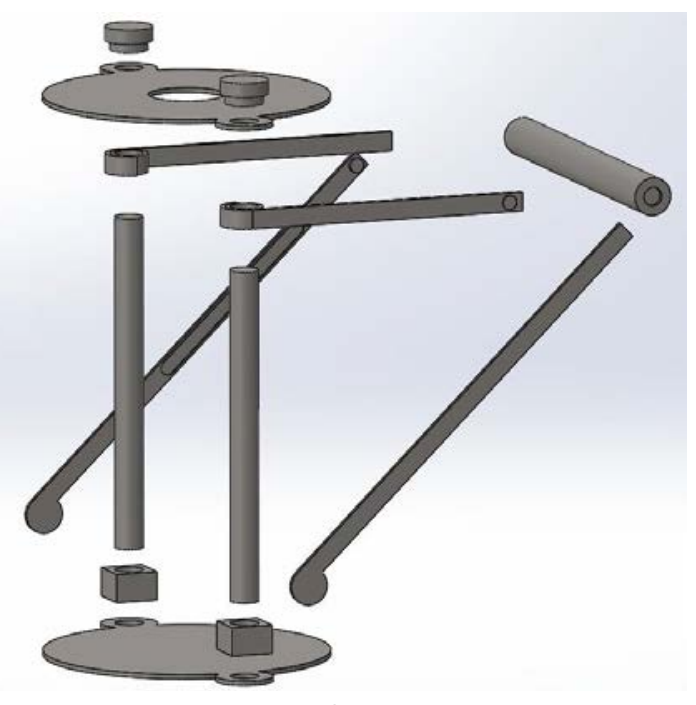

a.

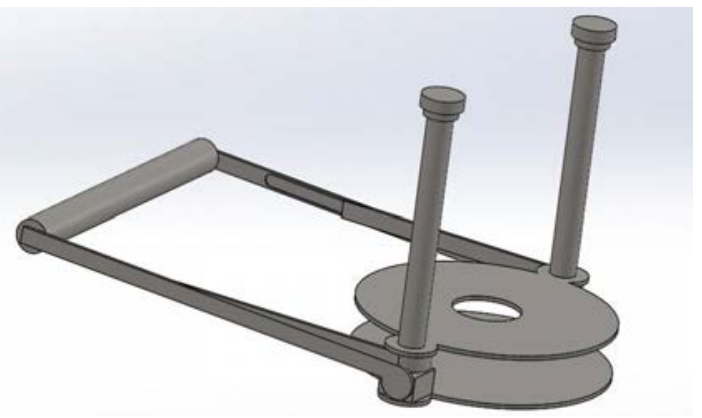

b.

Fig. 3 Geometric components of a mechanical Compactor PET (before assembly)

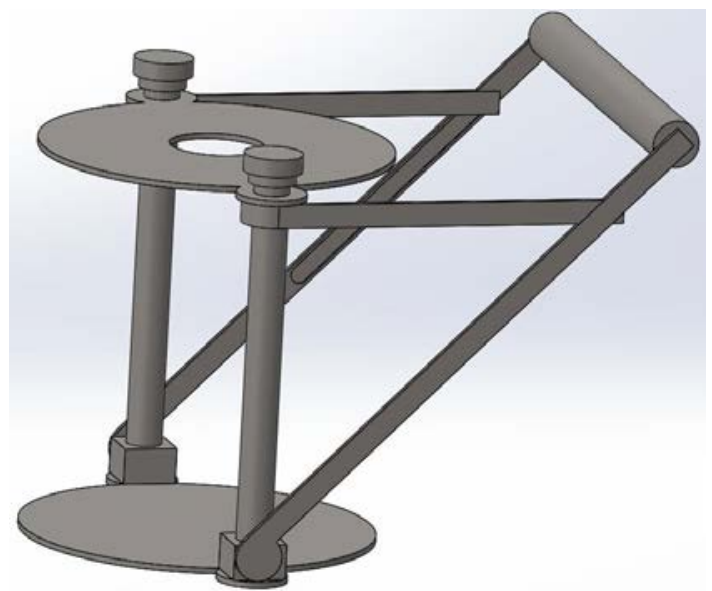

Fig. 4 Mechanical Compactor design for PET bottles 
In figures 5 are presented different types of PET bottles.

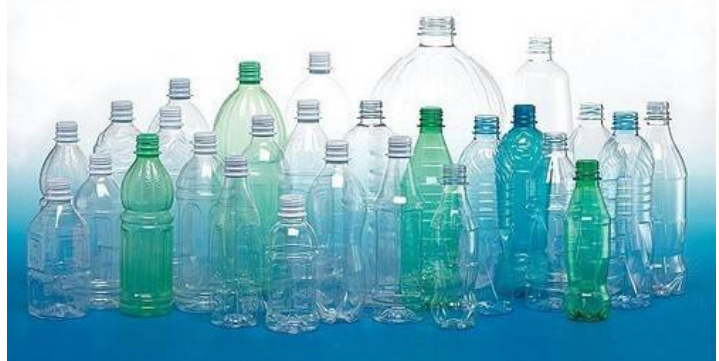

Fig. 5 Different types of PET bottles [4]

In table 1 are presented the volume of different types of PET bottles.

Table 1- Volume of different types of PET

\begin{tabular}{|l|l|l|l|l|l|}
\hline $\begin{array}{l}\text { PET } \\
\text { types }\end{array}$ & $\begin{array}{l}\text { PET } \\
2.51\end{array}$ & $\begin{array}{l}\text { PET } \\
21\end{array}$ & $\begin{array}{l}\text { PET } \\
1.51\end{array}$ & $\begin{array}{l}\text { PET } \\
11\end{array}$ & $\begin{array}{l}\text { PET } \\
0.51\end{array}$ \\
\hline Volume & $2.5 \mathrm{dm}^{3}$ & $2 \mathrm{dm}^{3}$ & $1.5 \mathrm{dm}^{3}$ & $1 \mathrm{dm}^{3}$ & $0.5 \mathrm{dm}^{3}$ \\
\hline
\end{tabular}

From the relations above it results that a PET bottle of 21 has $0.002 \mathrm{~m}^{3}$. This means that 1000 bottles of 21 take up a space of $2 \mathrm{~m}^{3}$.

With this compactor the space that the bottles will take to store will be reduced to almost $20 \%$. From 2 $\mathrm{m}^{3}$ it will go down to $0.4 \mathrm{~m}^{3}$.

The process of using the compactor is illustrated in figure 6.

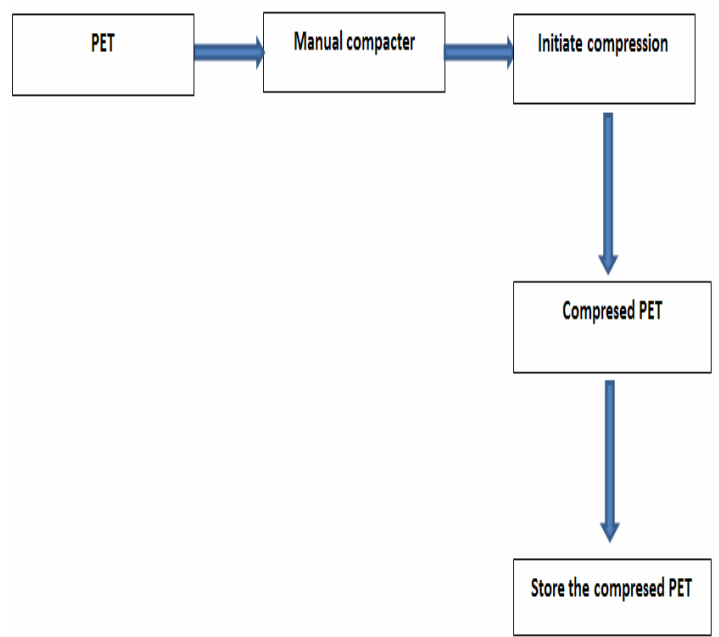

Fig. 6 The process of using the mechanical compactor for PET bottles

\section{CONCLUSION}

The advantages of products made from recycled plastic consist of cheaper manufacturing costs than raw materials.
Approximately $30 \%$ of the plastic produced is used for packaging. PET bottles are not biodegradable. The increase of their consumption, especially over the last 10 years, has led to alarming increases in the number of packages that are irresponsibly disposed of in nature.

By collecting and recycling them, is reduced the negative impact on the environment.

PET is, however, a very important issue for our country due to its low recycling rate. PET takes hundreds of years to degrade and it is a great resource consumer: all plastic production consumes $8 \%$ of world oil production. Recycling is therefore beneficial: recycling one ton of plastic saves 1.8 tons of oil and gains 6 cubic meters of storage space at the landfill. A single recycled plastic bottle saves the energy required for keeping an electric light bulb working for six hours.

From PET, we can produce clean flakes of plastic, which can be exported as they are (it usually costs around 300-400 Euro/ton depending on their quality) and they can be delivered to the industries that use them to create their products.

We must mention some relevant aspects of what we can do with plastic:

- 20 bottles of PET weigh around $1 \mathrm{~kg}$;

- 5 bottles of 2 liters are enough to make fibers for a big shirt;

- 20 bottles of 2 liters are enough for filling a winter jacket;

- 20 bottles of 2 liters are required for manufacturing a filter;

- 35 bottles of 2 liters are required for making a sleeping bag.

\section{REFERENCES}

[1] Dobre D., Dezvoltare de produs - Baze teoretice și studii de $c a z$, Ed. Bren, ISBN 97876067610711772 , București, 2014

[2] Goanță A.M., "Comparative study on the applicability of "extrusion" command in the design software taught in higher education institutions", The Annals of "Dunarea de Jos" University of Galati, Fascicle XIV, Mechanical Engineering, ISSN 1224-5615, pp.6-10, 2012.

[3] Goanță A.M., "Considerations on design controls intended to achieve thin walled bodies". The Annals of "Dunarea de Jos" University of Galati, Fascicle XIV, Mechanical Engineering, Issue vol. 2, ISSN 1224-5615, pp. 9-12., 2013.

[4] https://warehousebizongo.wordpress.com/2016/01/07/petbottle-industry-an-overview/

[5] http://sigurec.ro/ro/despre-sigurec/reciclez1/reciclarea-pet--urilor.html

[6] http://www.ecoteca.ro/ce-putem-face-din-pet-uri.htm

[7] https://www.amazon.com/Manual-PET-Bottle-PressEcopress/dp/B003UMIJ 8 U

[8] http://www.mase-plastice.ro/dictionar/p/pet.html

[9] https://news.un.org/en/story/2014/06/471492-plastic-wastecauses-13-billion-annual-damage-marine-ecosystems-says-unagency

[10] https://ro.wikipedia.org/wiki/Aluminiu 